\section{Intracranial pressure monitoring in a group of critically ill children}

Sir,

The article by Tasker et al describes their experience of intracranial pressure monitoring in a heterogeneous group of critically ill children. ${ }^{1}$ While agreeing with the principle of early intervention we wish to make several points.

Using almost exclusively the intraventricular catheter method of monitoring intracranial pressure described by McWilliam and Stephenson ${ }^{2}{ }^{3}$ we have managed 48 children aged 7 weeks to 13 years with a variety of disorders over the past five years (table).

\section{Table Details of the 48 children managed}

I Infectious and parainfectious illness Meningococcal disease Pneumococcal meningitis

Haemophilus influenzae meningitis Measles encephalitis

Mycoplasma encephalitis

Epstein-Barr virus encephalitis

Parainfectious encephalopathy

II Metabolic illness

Reye's syndrome ('idiopathic')

Medium chain acyl CoA dehydrogenase deficiency

Ornithine transcarbamylase deficiency

Diabetic hyperosmolar coma

III Hypoxic ischaemic encephalopathy

Sudden infant death syndrome, postcardiac arrest 2

Hydrocephalus, postrespiratory arrest

IV Haemolytic uraemic syndrome

V Haemorrhagic shock encephalopathy

VI Miscellaneous

Brain stem astrocytoma

Unexplained encephalopathy

Pseudomembranous colitis with septic shock

Total 48

*Failed technique.

Our technique for intraventricular pressure monitoring is simple and can be done by the physician at the bedside. In only one case did monitoring fail (failure rate $2 \%$ ). There was one case of secondary sepsis and no long term sequelae. There were no intracerebral haemorrhages. The only major difference in our management from that of the authors was that we used continuous infusions of morphine and pancuronium rather than bolus doses.

Twenty seven of our patients had a good or moderate neurological outcome and four were severely handicapped using the Seshia scale of assessment. ${ }^{4}$ Seventeen patients died. These results are very similar to those of the authors. The average cerebral perfusion pressure of our normal survivors, however, was $36.5 \mathrm{~mm} \mathrm{Hg}$, and nine of these 18 normal survivors had minimum cerebral perfusion pressure of less than $40 \mathrm{~mm} \mathrm{Hg}$. The duration of the minimum cerebral perfusion pressure could not be assessed as only hourly observations were charted by the nursing staff (in addition to the acute changes in cerebral perfusion pressure). Three of the 17 who died had a minimum cerebral perfusion pressure of greater than $45 \mathrm{~mm} \mathrm{Hg}$, but the average minimum of those who died was $20.7 \mathrm{~mm} \mathrm{Hg}$.

The difference was significant ( $\mathrm{p}$ less than 0.05 by paired $t$ test). In contrast with the authors' experience a child with Reye's syndrome who had maximum intracranial pressure of $65 \mathrm{~mm} \mathrm{Hg}$ survived to be normal. Eight of our survivors had a Glasgow coma scale of less than 5 (not attributable to drugs) before monitoring of intracranial pressure.

There are several areas of intracranial pressure monitoring which need further clarification. The question of what is an adequate cerebral perfusion pressure has yet to be resolved. Factors such as regional blood flow variation, duration of minimum cerebral perfusion pressure, and the underlying disease process may be of great importance.

We feel that taking a cerebral perfusion pressure of 40 $\mathrm{mm} \mathrm{Hg}$ as a cut off point without regard to the underlying illness and the duration of that pressure is at present wrong, and that more prospective research is needed in this area. Further, with our experience we would suggest that a Glasgow coma scale at less than 5 is not necessarily an indicator of a poor outcome. Therefore, neither of these parameters should necessarily be used to decide on the initiation or continuation of a treatment.

Finally, it is not clear how many of the patients of Tasker et al were transferred from other hospitals before the institution of neurointensive care. Over the past six months we have provided an intensive care transport service for critically ill children. During that period we have been asked to transport four children in whom the intracranial pressure was definitely raised or impaired cerebral perfusion pressure was suspected. In all cases the children were assessed by one of the authors and measures taken to reduce intracranial pressure and to stabilise mean arterial pressure before transfer from the referring hospital. We feel that in this way fluctuations in cerebral perfusion pressure may be minimised. Identifying illnesses in which cerebral perfusion pressure is significantly reduced and developing scoring systems allowing timely intervention may minimise later handicap.

\section{References}

1 Tasker RC, Matthew DJ, Helms P, Dinwiddie R, Boyd S. Monitoring in non-traumatic coma. Part I: invasive intracranial measurements. Arch Dis Child 1988;63:888-94.

2 McWilliam RC, Stephenson JBP. Rapid bedside technique for intracranial pressure monitoring. Lancet 1984;ii:73-5.

3 McWilliam RC, Stephenson JBP. Bedside intracranial pressure monitoring. Lancet 1985;ii:341.

${ }^{4}$ Seshia SS, Seschia MMK, Sachdeva RK. Coma in childhood. Dev Med Child Neurol 1977;19:614-28.

G Balakrishnan, C H Skeoch, J B P Stephenson, R C McWilliam, D Hallworth, and J F Sinclair Royal Hospital for Sick Children, Yorkhill, Glasgow G3 8SJ 